\title{
The effect of bone marrow mesenchymal stem cell and nano-hydroxyapatite/collagen I/poly-L-lactic acid scaffold implantation on the treatment of avascular necrosis of the femoral head in rabbits
}

\author{
LE WANG ${ }^{1}$, LEIXIN XU ${ }^{2}$, CHANGLIANG PENG ${ }^{1}$, GUOXIN TENG $^{3}$, \\ $\mathrm{YU} \mathrm{WANG}^{4}$, XIAOSHUAI XIE ${ }^{5}$ and DONGJIN WU ${ }^{1}$ \\ ${ }^{1}$ Department of Spinal Surgery, Second Hospital of Shandong University, Jinan, Shandong $250033 ;{ }^{2}$ Department of \\ Orthopedics, The Fourth People's Hospital, Heze, Shandong 274100; ${ }^{3}$ Department of Pathology, Second Hospital of \\ Shandong University, Jinan, Shandong 250033; ${ }^{4}$ Department of Radiology, Affiliated Hospital of Shandong \\ Traditional Chinese Medicine University, Jinan, Shandong 250012; ${ }^{5}$ Department of Kidney Transplantation, \\ Second Hospital of Shandong University, Jinan, Shandong 250033, P.R. China
}

Received April 24, 2019; Accepted June 20, 2019

DOI: $10.3892 /$ etm.2019.7800

\begin{abstract}
For avascular necrosis of the femoral head (ANFH), repair and regeneration are difficult because of the edema and high pressure caused by continuous ischemia and hypoxia. Core decompression (CD) is a classic method for treating early ANFH before the collapse of the femoral head; however, its effect is still controversial. To improve the therapeutic effect of CD on ANFH, a novel tissue-engineered bone (TEB) was constructed by combining bonemarrow mesenchymal stemcells (BMSCs) with nano-hydroxyapatite/collagen I/poly-L-lactic acid (nHAC/PLA) scaffolds and implanting the TEB into the bone tunnel of CD. Cell attachment was observed by scanning electron microscopy and hematoxylin and eosin staining. The authors' previous studies confirmed that nHAC/PLA is an excellent scaffold material with favorable biocompatibility and no cytotoxicity. A total of 24 New Zealand rabbits with ANFH were randomly divided into three groups, as follows: Group A ( $n=8)$, pure CD; group B $(n=8), C D+n H A C / P L A ;$ and group $\mathrm{C}(\mathrm{n}=8), \mathrm{CD}+\mathrm{BMSCs}-\mathrm{nHAC} / \mathrm{PLA}$. The favorable effect of BMSCs-nHAC/PLA on angiogenesis and bone formation in necrotic areas was further evaluated via radiographic and histological analyses. Computerized tomography (CT) scanning and $\mathrm{H} \& \mathrm{E}$ staining showed more capillaries and new osteoid tissue in group $\mathrm{C}$ compared with in groups $\mathrm{B}$ and $\mathrm{A}$.
\end{abstract}

Correspondence to: Dr Dongjin Wu, Department of Spinal Surgery, Second Hospital of Shandong University, 247 Bei Yuan Street, Jinan, Shandong 250033, P.R. China

E-mail: doctorwdj@163.com

Key words: bone marrow mesenchymal stem cells, cell scaffold, tissue engineered bone, avascular necrosis of femoral head, core decompression
Micro-CT showed that the new bone coverage rate and implanted material degradation degree were each increased in group $\mathrm{C}$ compared with in group $\mathrm{B}$. These results indicate that BMSCs-nHAC/PLA scaffolds may improve the curative effect of $\mathrm{CD}$ and provide a strategy for treating ANFH.

\section{Introduction}

Avascular necrosis of the femoral head (ANFH) is a common orthopedic disease that is caused by alcohol abuse, long-term use of hormones and trauma (1-3). It has a high incidence, difficult early diagnosis and prevention, and core decompression (CD) can only stop the progression of the disease $(4,5)$. Determining how to promote regeneration and repair in ANFH, especially in young patients, and how to further reduce or even avoid total hip arthroplasty has attracted extensive attention from researchers (6). Previous studies have reported that CD combined with autogenous bone transplantation has a certain therapeutic effect $(2,7)$; however, it makes patients suffer more trauma and carries the risk of there being insufficient autogenous bone. Therefore, the present study hopes to find a method to reverse necrosis and promote tissue regeneration and repair through $\mathrm{CD}$ and implantation of tissue-engineered bone (TEB).

BMSCs are an ideal candidate for tissue engineering because the cells are easily isolated and cultured and have rich differentiation potential $(8,9)$. Scaffold materials with $3 \mathrm{D}$ micropore structures can promote the growth of neovascularization and induce MSC differentiation to enhance osteogenesis at the bone defect site $(10,11)$. A 3D porous biomimetic scaffold, nano-hydroxyapatite/collagen I/poly-L-lactic acid (nHAC/PLA), with properties similar to natural bone, has drawn increasing attention (12). The authors' previous study demonstrated that nHAC/PLA has excellent biocompatibility, osteoconduction and biodegradability when used to repair long bone defects (13). CD combined with stem cell or bone substitute transplantation has been reported in animal and 
clinical studies, but still has not achieved high tissue regeneration and repair efficiency $(7,14)$.

In the present study, bone marrow mesenchymal stem cells (BMSCs) were first successfully inoculated into nHAC/PLA scaffolds and then implanted the scaffolds into $\mathrm{CD}$ bone tunnels in rabbits. All results of radiological and histological examination confirmed that BMSCs-nHAC/PLA scaffold implantation can effectively promote the therapeutic effect of $\mathrm{CD}$ for ANFH, supporting the pursuit of BMSCs-nHAC/PLA scaffolds as a new treatment for ANFH.

\section{Materials and methods}

BMSC culture. The current study was carried out in accordance with the Guidelines on the Care and Use of Laboratory Animals issued by the Animal Research Council of China (15) and was approved by the Animal Research Ethics Committee of the Second Hospital of Shandong University. Fig. 1 shows a brief flow of modeling, grouping and experimentation. At the same time, the bone coverage and microvessel density of each group were compared.

Primary high-purity rabbit BMSCs, confirmed by directional differentiation and phenotypic identification, were provided by Dr Xiaoshuai Xie (Second Hospital of Shandong University) (16). BMSCs were resuspended in 89\% Dulbecco's modified Eagle's medium (Gibco; Thermo Fisher Scientific, Inc.), $10 \%$ fetal bovine serum (HyClone; GE Healthcare) and $1 \%$ penicillin/streptomycin/amphotericin B (Sangon Biotech, Co., Ltd.). Then, cells were cultured at $37^{\circ} \mathrm{C}$ with $5 \% \mathrm{CO}_{2}$ and $95 \%$ humidity. The culture medium was changed every 2 days. Cells were passaged when they reached $80-90 \%$ confluence and used for subsequent experiments at passage 3 .

BMSC-seeded scaffolds. Briefly, the 2- $\mathrm{mm}^{3} \mathrm{nHAC} / \mathrm{PLA}$ scaffolds (Beijing Allgens Medical Science \& Technology Co., Ltd.) were pre-wetted overnight and placed in a 96-well culture plate (1/well). Then, $5 \times 10^{5}$ cells/ml $(100 \mu \mathrm{l})$ were injected into a stent using a $29 \mathrm{G}$ fine needle (Ultra-Fine; Becton-Dickinson and Company). Then, $100 \mu \mathrm{l}$ culture medium was added to each well and replaced every $6 \mathrm{~h}$.

BMSC attachment to scaffolds. The morphology and quantity of BMSCs in the scaffolds was assessed by hematoxylin and eosin (H\&E) staining under light microscopy at a magnification of x100 and scanning electron microscopy (SEM; Hitachi SU8010; Hitachi, Ltd.) at a magnification of x1,000 after $24 \mathrm{~h}$. Simply, the BMSCs-nHAC/PLA scaffolds were rinsed three times with phosphate-buffered saline ( $\mathrm{PBS} ; \mathrm{pH}=7.4)$, fixed in $4 \%$ paraformaldehyde for 2 days at $4^{\circ} \mathrm{C}$, decalcified in $10 \%$ EDTA and embedded in paraffin. Then, the samples were cut to a $5-\mu \mathrm{m}$ thickness and stained with hematoxylin for $3 \mathrm{~min}$ and $30 \mathrm{sec}$, and water-soluble eosin for $2 \mathrm{~min}$ and $30 \mathrm{sec}$ at $35^{\circ} \mathrm{C}$. The rinsed BMSCs-nHAC/PLA scaffolds were then fixed in $2.5 \%$ glutaraldehyde for $12 \mathrm{~h}$ at $4^{\circ} \mathrm{C}$, dehydrated in graded ethanol, dried at the critical point and sputter coated with gold for SEM examination.

Animal model. A total of 30 healthy New Zealand rabbits (age, 2 months old; weight, 1.5-2.5 kg; male:female ratio, 1:1) were purchased from the Animal Laboratory Center of the
Second Hospital of Shandong University and used to establish ANFH models. The rabbits were housed at $24^{\circ} \mathrm{C}$, with $50 \%$ humidity, a light and dark cycle of $12 \mathrm{~h} / 12 \mathrm{~h}$ and free access to food and water in a single cage, according to the protocols outlined in a previous study (9). First, lipopolysaccharide (LPS; $10 \mu \mathrm{g} / \mathrm{kg}$; Sigma-Aldrich; Merck KGaA) was implemented intravenously on day 1 and then methylprednisolone acetate $(20 \mathrm{mg} / \mathrm{kg}$; Pfizer, Inc.) was administered intramuscularly into the right gluteus medius muscle on days 2, 3 and 4. A total of three rabbits died of pulmonary infection within 2 weeks of model establishment. Three rabbits were randomly sacrificed by intravenous injection of an overdose of sodium pentobarbital solution $(100 \mathrm{mg} / \mathrm{kg})$ to verify ANFH using magnetic resonance imaging, conducted by two experts from the Department of Radiology, Affiliated Hospital of Shandong Traditional Chinese Medicine University, at 6 weeks after the last injection of methylprednisolone (MPSL).

$C D$ and BMSCs-nHAC/PLA implantation. The rabbits with ANFH were randomly divided into 3 groups, as follows: Group A ( $n=8)$, pure CD; group B $(n=8), C D+n H A C / P L A$; and group $\mathrm{C}(\mathrm{n}=8), \mathrm{CD}+\mathrm{BMSCs}-\mathrm{nHAC} / \mathrm{PLA}$. All rabbits were anesthetized by ear vein injection of pentobarbital sodium (30 mg/kg). A $2 \mathrm{~cm}$ incision was made from the femoral greater trochanter of the right hind limb to the hip and the posterior lateral capsule of the hip joint was opened. A 3-mm surgical drill bit was used to create a $3 \times 4 \mathrm{~mm}$ decompression tunnel from the junction of cartilage and bone to the medullary core, then the $\mathrm{nHAC} / \mathrm{PLA}$ scaffolds $\left(2 \times 2 \mathrm{~mm}^{3}\right.$, group B) and BMSCs-nHAC/PLA scaffolds $\left(2 \times 2 \mathrm{~mm}^{3}\right.$, group C) were placed into the bone tunnels by fit pressure (Fig. 2). Finally, the incision was stitched down layer by layer. After the operation, the animals received the same diet in single cage in a clean, dry and well-ventilated animal house.

Radiology and histology analyses. At 4 weeks post-surgery, rabbits were sacrificed using the same procedure as that for establishing ANFH models described above for CT (General Electric Company; groups A-C) and micro-CT (Sky Scan; Bruker Corporation; groups B and C). New bone around the decompression tunnel and implants in the tunnel were observed, and the bone coverage was calculated automatically by CT-An analysis software (version 1.15.4.0; Bruker Corporation). Then, to further observe histopathological changes in the bone tunnel area, the femoral heads of each group were stained with H\&E, as described above. The new bone, vascular tissue and implant degradation were evaluated by two histopathology specialists from the Second Hospital of Shandong University.

Statistical analyses. Each experiment was repeated three times. The data obtained are expressed as the mean \pm standard deviation. The differences between groups were analyzed using an unpaired two-sided Student's t-test when only two groups, or by one-way analysis of variance followed by post hoc Bonferroni's tests when more than two groups were compared. All analyses were performed with SPSS version 18.0 (SPSS, Inc.). $\mathrm{P}<0.05$ was considered to indicate a statistically significant difference. 


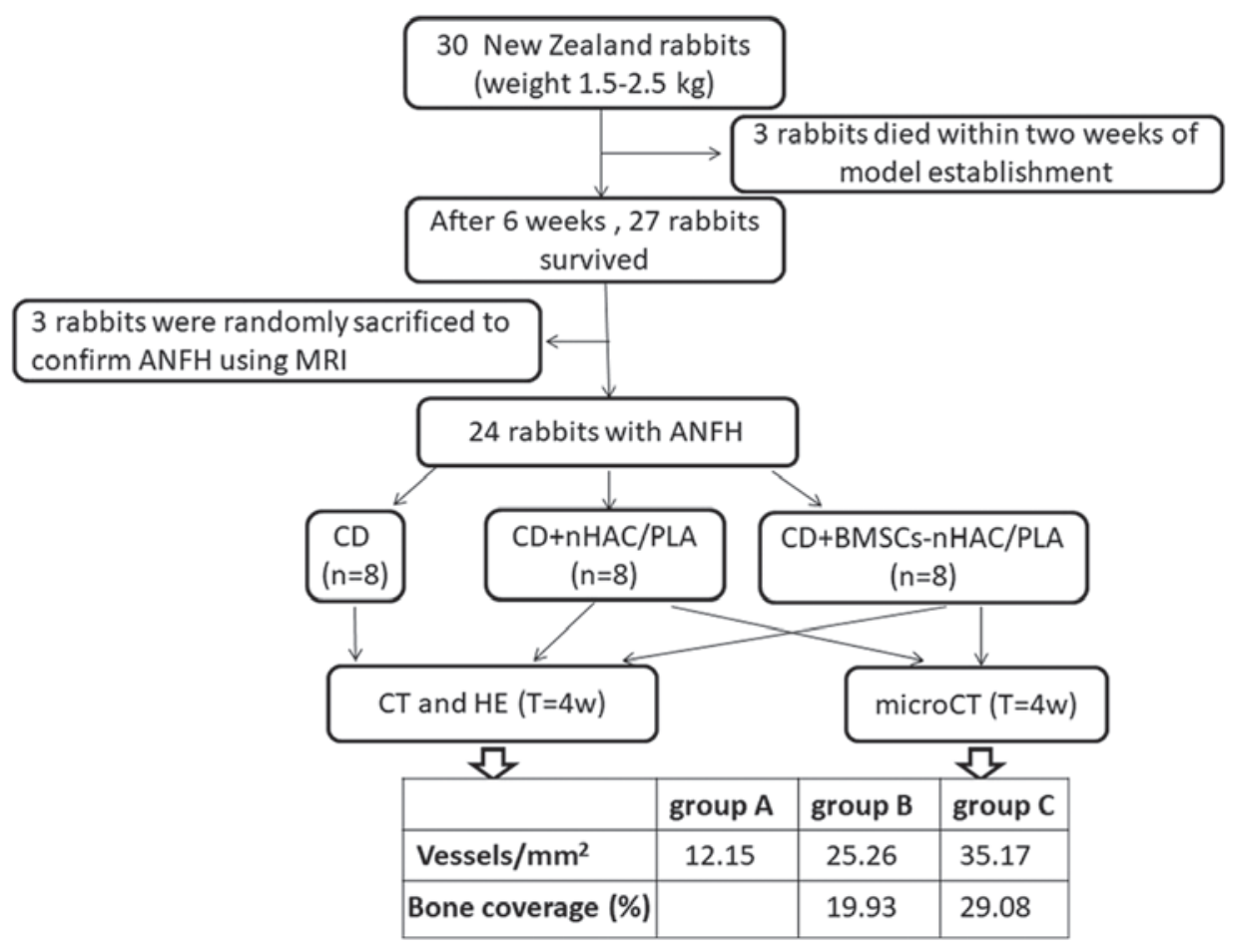

Figure 1. General flow chart of the experiment and some experimental results.
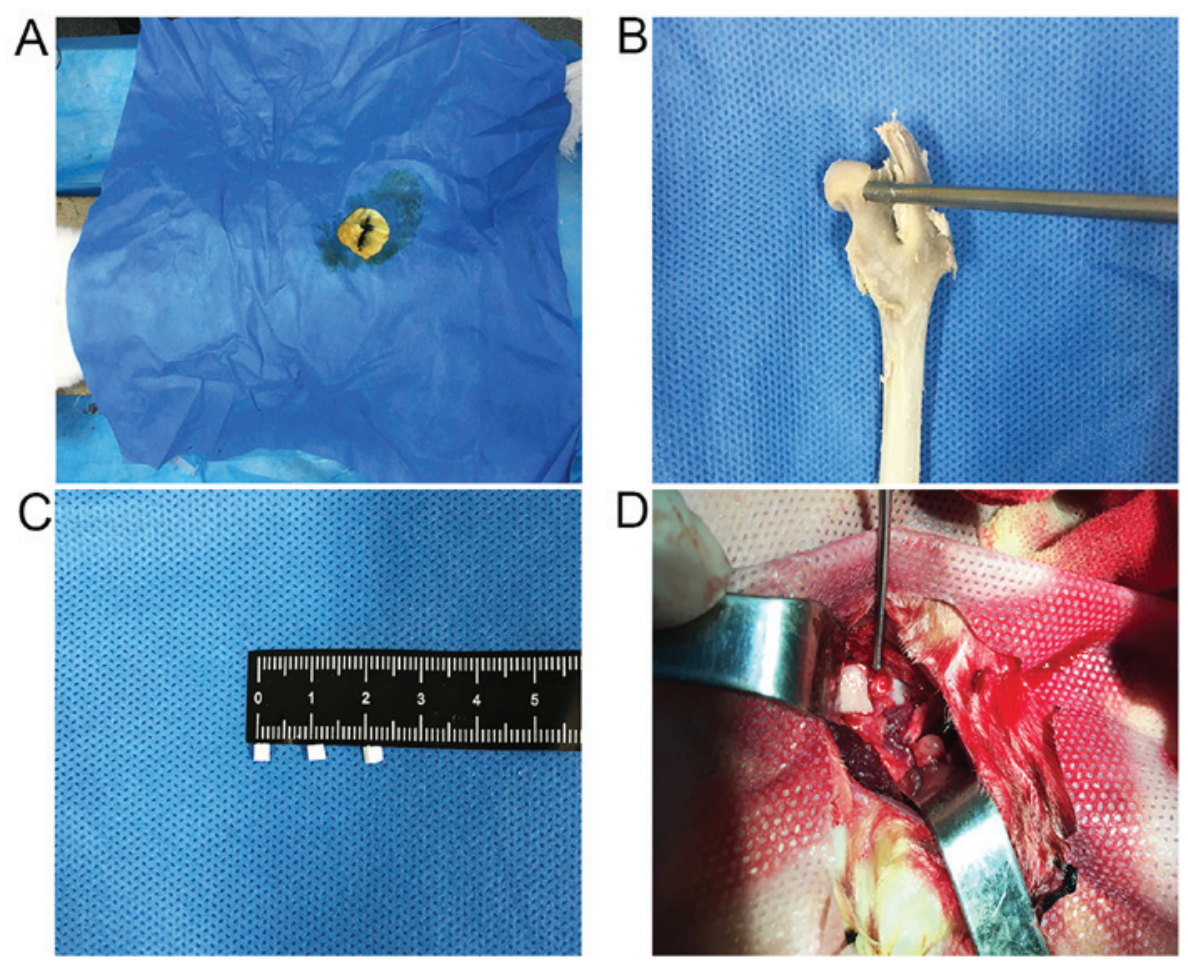

Figure 2. CD and graft filling. (A) After successful anesthesia, the incision was marked, the operative area was disinfected and the sterile opening was covered with a towel. (B) Demonstration of the CD approach to the femoral head in vitro. (C) nHAC/PLA scaffold size measurement (2 $\left.\mathrm{mm}^{3}\right)$. (D) nHAC/PLA or BMSCs-nHAC/PLA were implanted into the decompression tunnel by fit pressure. CD, core decompression; BMSC, bone marrow stem cells; nHAC/PLA, nano-hydroxyapatite/collagen I/poly-L-lactic acid; H\&E, hematoxylin and eosin; CT, computed tomography; ANFH, avascular necrosis of the femoral head; MRI, magnetic resonance imaging.

\section{Results}

Cell culture. To observe and evaluate the growth and morphology of BMSCs, the cell phenotype of which was identified by Dr Xiaoshuai Xie (16). Primary rabbit BMSCs were incubated in $25-\mathrm{cm}^{2}$ plastic tissue culture flasks. On day 1 , all cells were adherent and mostly spindle-shaped, though a few irregular cells were triangular or rhomboid. At 

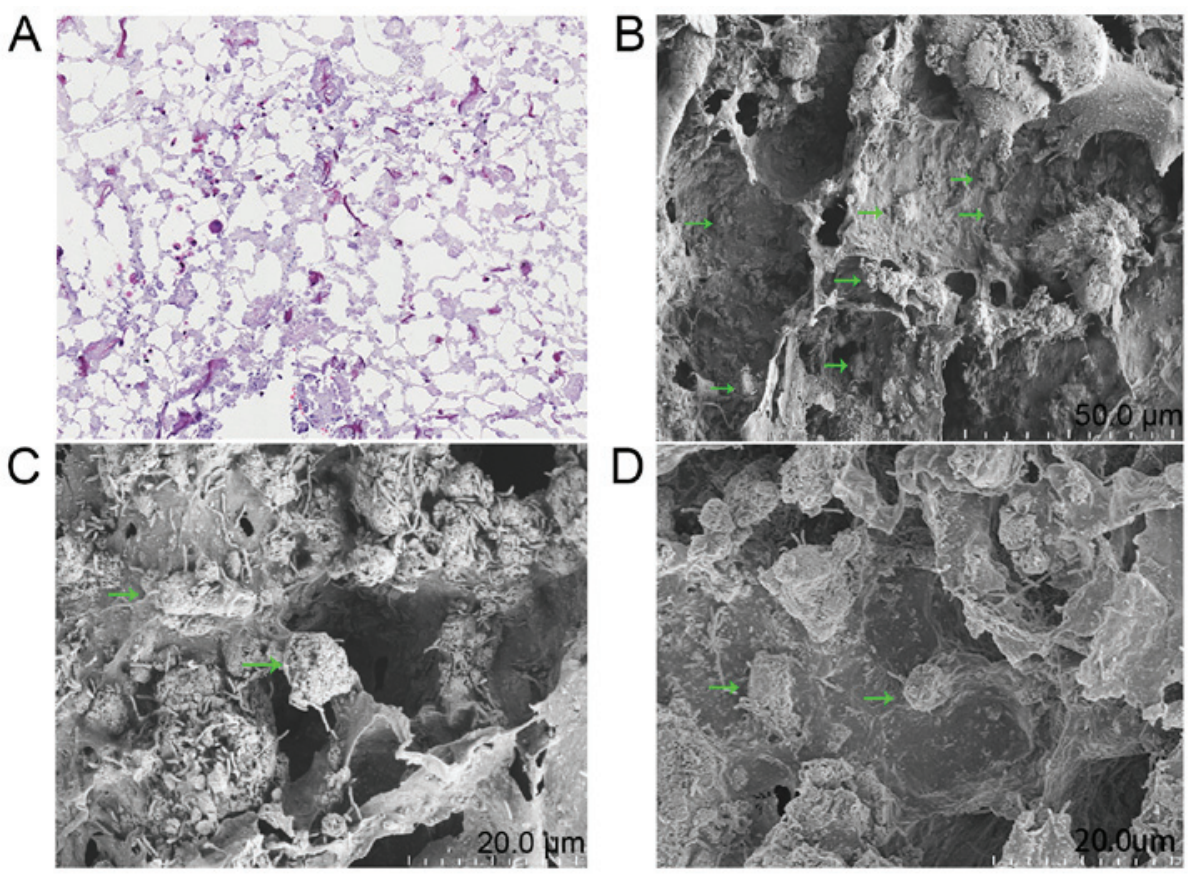

Figure 3. BMSCs could attach to scaffolds. (A) A total of $24 \mathrm{~h}$ after seeding, hematoxylin and eosin staining showed that a large number of BMSCs adhered to the scaffold and were distributed evenly (x200). (B) Scanning electron microscopy micrographs revealed that BMSCs were uniformly distributed inside the scaffold; the cell bodies and filamentous pseudopodia contacted the micropore wall. (C) The bodies of the poorly attached cells were spherical in shape and only two to three short and thick pseudopods contacted the inner surface of the scaffold. (D) The adherent cell bodies were long and oval-shaped, or spherical. One side of which was closely attached to the pore wall of the scaffold and many banded pseudopodia radiated from it (denoted by the green arrowhead). BMSCs, bone marrow stem cells.

day 5 , the cells reached $80-90 \%$ confluence. These findings are consistent with those reported by Xie et al (16), confirming that well-grown purified BMSCs were obtained.

Scaffold and cell attachment. To assess the 3D microstructure of nHAC/PLA, SEM was used to verify the size and direction of the stent micropores. The results are similar to those reported before; the pore sizes are $\sim 300 \pm 250 \mu \mathrm{m}$, with a porosity of $70-90 \%$ (12). The number of cells in the scaffold was evaluated by $H \& E$ staining under light microscopy and the micro-morphology of adherent cells was further observed by SEM, which confirmed that the cells was firmly attached to the scaffold. At $24 \mathrm{~h}$, a number of spindle cells were uniformly distributed inside the scaffold; the cell bodies and filamentous pseudopodia contacted the micropore wall. The adherent cell bodies were long and oval, one side of which was closely attached to the pore wall of the scaffold, and a number of banded pseudopodia radiated from it. The bodies of the poorly attached cells were spherical in shape and only 2-3 short and thick pseudopods contacted the inner surface of the scaffold (Fig. 3). Using SEM, similar were found results compared with the authors' previous studies $(10,13,17)$. These results confirm that the $3 \mathrm{D}$ inoculation of cells into scaffolds can be accomplished by multi-point injection via fine needles.

$C D$ and general condition. The present study aimed to not only avoid radiation exposure, but also to complete the operation quickly and accurately. In the process, without X-rayed guiding, all surgical operations were completed in 20-30 min, with an incision of $\sim 2 \mathrm{~cm}$ and intraoperative bleeding of 3-5 ml. All rabbits were awake 1-2 h post-surgery. The diet and behavior of the rabbits had returned to normal by 3 days after surgery. Within 4 weeks post-surgery, no rabbits developed femoral fractures or hip dislocation. Despite being without antibiotic use, all rabbits exhibited no obvious wound infection after 4 weeks. These results proved that the posterolateral subchondral approach, without X-rayed guiding, could quickly and accurately complete the $\mathrm{CD}$, avoiding radiation exposure of the operator, and was conducive to the rapid recovery of experimental animals after surgery.

Radiology analyses. To observe osteogenesis in the early decompression tunnel, all rabbits were examined by $\mathrm{CT}$ at 4 weeks after the operation and bone formation and scaffold degradation in the $\mathrm{B}$ and $\mathrm{C}$ groups were observed more intuitively by micro-CT. Via CT test, group C showed a homogeneous high-density signal in the decompression tunnel and the outlet of the tunnel was closed. In group B, the first half of the decompression tunnel shows an inhomogeneous high-density signal; the second half shows an inhomogeneous low-density signal and the tunnel opening was not closed. However, in group A, the entire tunnel shows an uneven low-density signal and there was no marked high-density signal in the decompression tunnel, and the external opening was not shut (Fig. 4). The micro-CT results further showed more bone trabeculae around the decompression tunnel in group C (Fig. 5A-C) relative to in group B (Fig. 5D-F); the trabeculae gradually spread to the center, making the tunnel narrow and irregular; the outlet was closed; and the implant was decomposed into multiple small pieces. The bone coverage percentages of groups B and C were 19.93 and $29.08 \%$, respectively and they were significantly different to each other $(\mathrm{P}<0.01$; Fig. $6 \mathrm{~A})$. These results confirmed that 


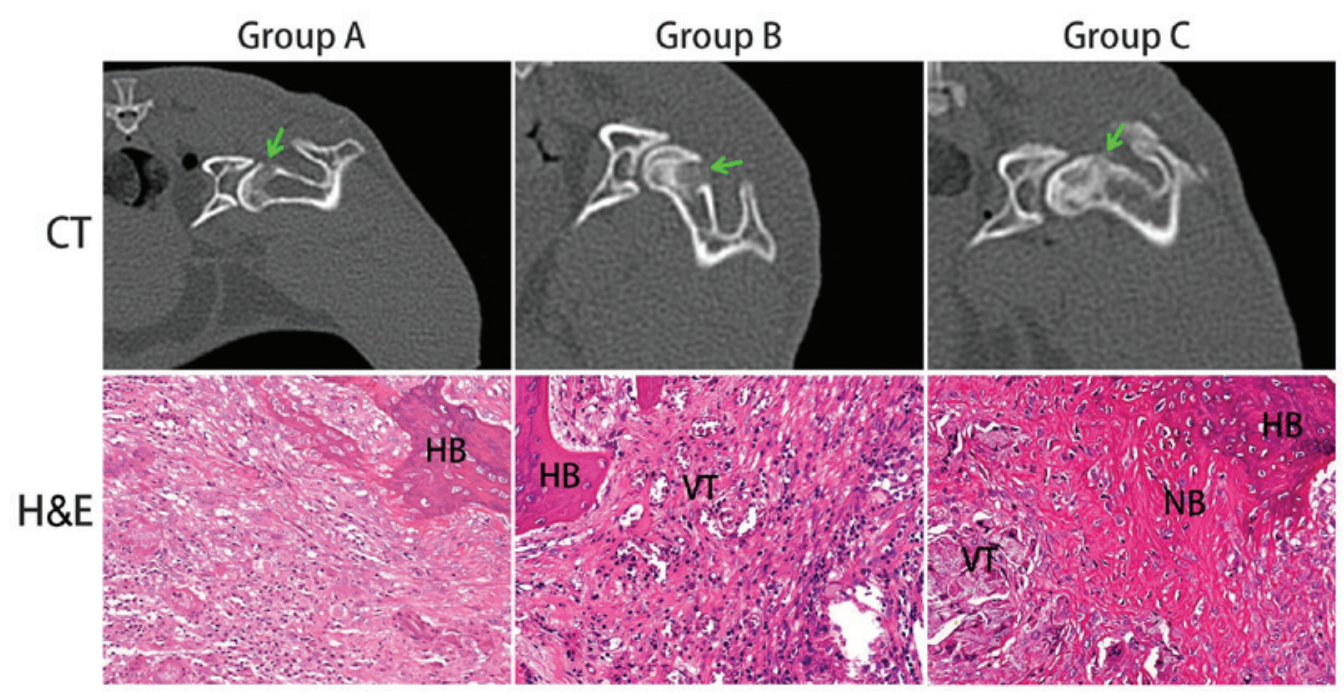

Figure 4. CT images and HE micrographs at 4 weeks post-operation. The CT images suggested that the osteogenesis in the decompression tunnel of group C was significantly higher than that in the other two groups. Histology micrographs of H\&E staining of bone tunnels (x200) of the three groups. CT, computerized tomographic scanning; NB, new bone; HB, host bone; VT, vascular tissue; H\&E, hematoxylin and eosin.

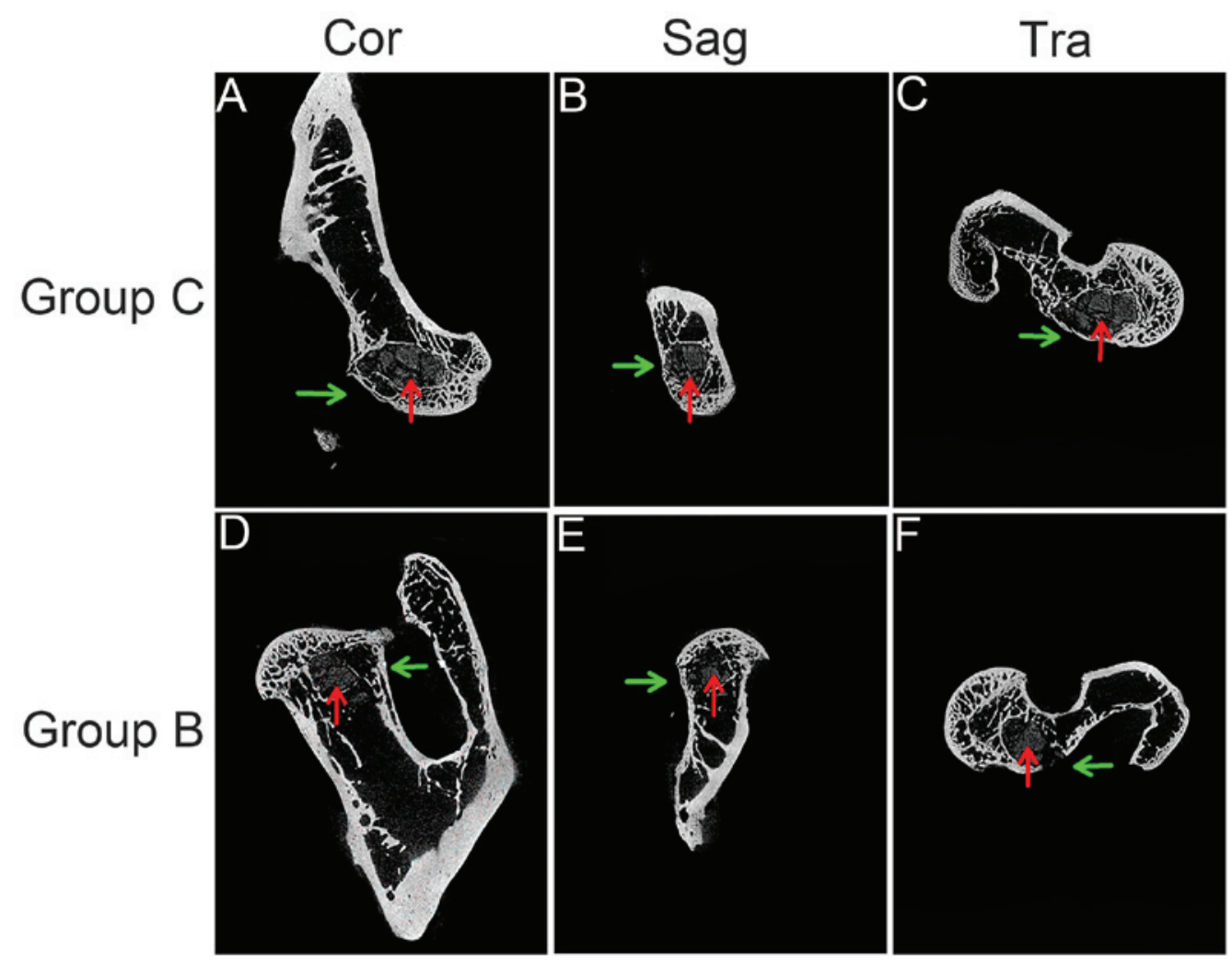

Figure 5. Micro-CT examination. (A) Cor, (B) Sag and (C) Tra micro-CT examination showed that the trabeculae around the subfemoral decompression tunnel in group $\mathrm{C}$ were compact and arranged regularly, the new bone gradually grew into the center of the tunnel, and tissue-engineered bone was wrapped well and degraded. At the same time, the exit of the tunnel was sealed by a thin layer of new bone. (D) Cor, (E) Sag and (F) Tra micro-CT examination showed that the trabeculae around the subfemoral decompression tunnel in group B were loose and disordered. No new bone was found in the inner wall and scaffold of the tunnel, and the degraded nHAC/PLA was not tightly wrapped by the new bone, and the exit of the tunnel was not sealed by the new bone (green arrows point to decompression tunnels and red arrows point to implants). CT, computerized tomography; Cor, coronal section; Sag, sagittal section; Tra, transverse section; nHAC/PLA, nano-hydroxyapatite/collagen I/poly-L-lactic acid.

CD combined with BMSCs-nHAC/PLA stent implantation had stronger osteogenic activity compared with the other two groups in the decompression tunnel and micro-CT showed that BMSCs-nHAC/PLA had better biodegradability than pure nHAC/PLA.
Histology analyses. To evaluate the angiogenesis of osteoid tissues in early bone tunnels, H\&E staining was performed on the decompression areas 4 weeks after the operation and counted the microvessel density by randomly selecting multiple visual fields under a microscope. In group $\mathrm{C}$, a large number of 

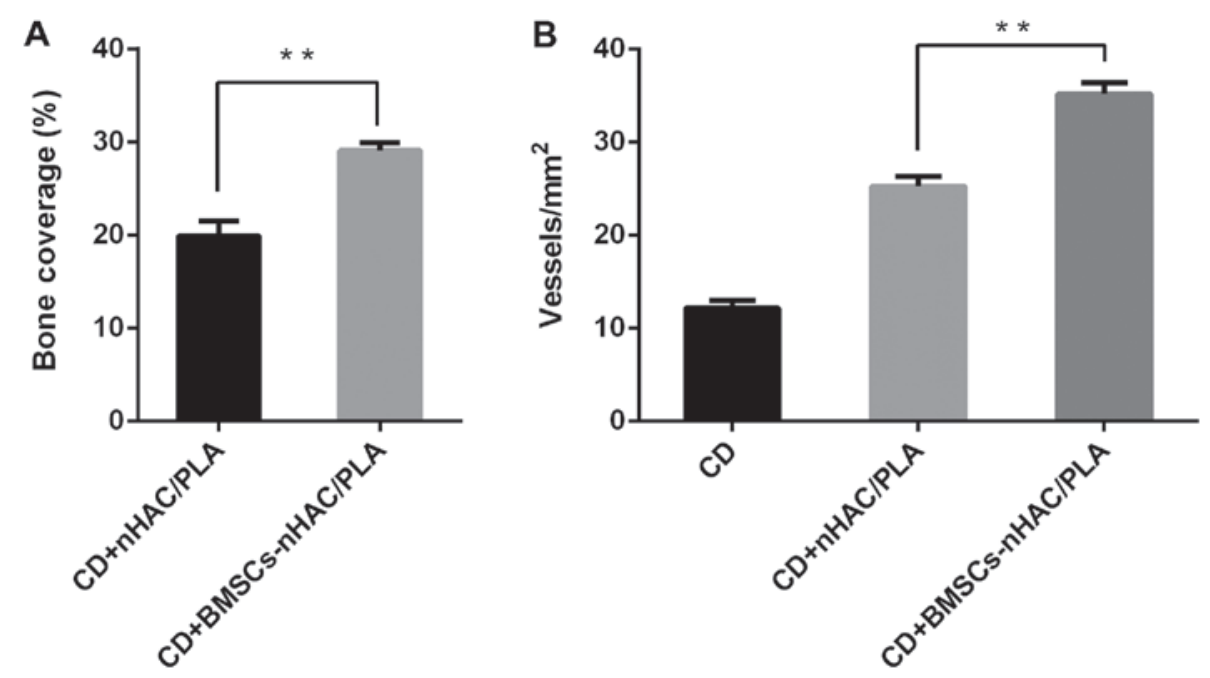

Figure 6. Bone coverage and microvascular density. (A) The percentage of bone coverage (group B and C) was measured at the experimental site. Data are presented as the mean $\pm \mathrm{SD}$. ${ }^{* *} \mathrm{P}<0.01$ according to the Student's two-sided unpaired t-tests. (B) The number of vessels at the experimental site (groups A, $\mathrm{B}$ and $\mathrm{C}$ ) was calculated. Data are presented as the mean $\pm \mathrm{SD}$. ${ }^{* *} \mathrm{P}<0.01$ according to one-way analysis of variance and post hoc Bonferroni's tests. nHAC/PLA, nano-hydroxyapatite/collagen I/poly-L-lactic acid; CD, core decompression.

new bones were connected to the host bone at the edge of the decompression tunnel. The scaffolds were divided into a number of islets by new osteoid tissue and abundant neovascularizations were observed around and inside the tunnel. Compared with group C, group B had similar changes at the edge of the tunnel, but fewer new bones, new osteoid tissues and blood vessels. Undecomposed stents were still observed in the center of the decompression tunnel and the distribution of new blood vessels in the stents was not obvious. However, in group A, almost no new bone or new vessels were observed (Fig. 4). Quantification of vessel densities showed that the neovascularization in group $\mathrm{C}$ (35.17 vessels $/ \mathrm{mm}^{2}$ ) was significantly increased compared with in groups B (25.26 vessels $\left./ \mathrm{mm}^{2}\right)$, and markedly increased compared with A (12.15 vessels $/ \mathrm{mm}^{2}$; P $<0.01$; Fig. 6B). These findings showed that BMSCs promoted angiogenesis in the inner wall of the tunnel and nHAC/PLA stent in the early stage of post operation, which was beneficial to the degradation of the stent and to tissue regeneration and repair.

\section{Discussion}

In the current study, TEB was constructed with BMSCs and nHAC/PLA and implanted into the bone tunnel created in CD. In vivo, BMSCs-nHAC/PLA promoted the regeneration of bone tissue and vascular tissue in the necrotic area, which may be beneficial for preventing or repairing ANFH.

BMSCs are one of the most commonly used cells in basic research and the clinical treatment of orthopedic diseases (18); BMSCs provide not only cell sources, but also cytokines and growth factors to accelerate the healing process of femoral head necrosis (19). PLA, hydroxyapatite (HA) and collagen are widely used in the manufacture of bionic artificial bone materials, which have achieved good results in clinical treatment (19). HA or collagen has the potential to promote the osteogenic differentiation of stem cells independently (20). Highly interconnected $3 \mathrm{D}$ microporous nHAC/PLA, which is a suitable scaffold for penetration by cells, organizes blood vessels to support growth and provides nutrients for regenerating tissue (21). When the volume of TEB is larger than $3 \mathrm{~mm}^{3}$, seeded cells have difficulty surviving due to the lack of a vascular network (22). Therefore, $2 \mathrm{~mm}^{3} \mathrm{nHAC} / \mathrm{PLA}$ was selected to produce TEB by seeding with BMSCs; this size ensures that cells on the surface and inside the scaffolds will have a nutrient and oxygen supply. During the construction of TEB, some researchers have directly dripped the cell suspension onto the scaffold surface and then performed co-culture $(12,17,21)$. It is difficult to uniformly distribute seed cells on the surface of and the inside of the scaffold. Most of the seed cells form a cell sheet covering the surface of the scaffold. Additionally, the self-propagation of cells in the scaffold requires long-term in vitro culture, which undoubtedly increases the TEB production time. In the present study, the cell suspension was injected into the scaffolds at different depths and points with a fine needle and then vertically dripped onto the surface of the scaffolds so that the seed cells were quickly and well distributed inside and outside the scaffolds. The experiment confirmed that, after 1 day, the cells were evenly distributed inside and outside the scaffold, and firmly attached.

The ANFH model, generated by combining LPS with a large dose of MPSL, does not require dislocation of the hip joint $(8,23)$; in contrast to liquid nitrogen freezing of the cartilage surface (24), early damage to blood flow and motor function of the femoral head was avoided. Unfortunately, three rabbits died in the preliminary stages of establishing the model. Previous studies $(9,25)$ have shown that the procedure for $\mathrm{CD}$ of the femoral head in rabbits usually involves drilling a bone tunnel from $1 \mathrm{~cm}$ below the femoral trochanter to the subchondral region of the femoral head through the femoral neck. This approach not only carries the risk of damaging the femoral neck and proximal lateral cortical bone, but it also exposes the model animals and the operators to radiation. In the present study, the posterolateral femoral head was exposed through a $2-\mathrm{cm}$ incision and a $3 \times 4 \mathrm{~mm}$ bone tunnel was drilled into the medullary center of the femoral head through the cartilage-bone junction. In this way, not only could the $\mathrm{CD}$ could be carried out accurately, but the risk of radiation exposure was also avoided. Additionally, by keeping bleeding 
and the operation time close to their estimates, all rabbits were restored to their pre-operative state, as determined by observing their post-operative diet and activities, at 2-3 days after operation without having been given prophylactic antibiotics. To a certain extent, the modified CD approach is fast, accurate and minimally invasive, which is conducive to rapid post-surgery recovery.

In the present study, it was confirmed that it is feasible to construct TEB by fine needle injection and decompression of the posterolateral approach to the femoral head to treat ANFH in rabbits. A total of four weeks after the operation, imaging and histological analyses showed more degradation of the implants in group C compared with in group B, and that the osteogenesis and vascularization activities in group $\mathrm{C}$ were better than those in groups $\mathrm{B}$ and $\mathrm{A}$. These results indicate that BMSCs-nHAC/PLA stent implantation can promote the repair and regeneration of a necrotic area and improve the therapeutic effect of CD on ANFH. BMSCs-nHAC/PLA stent implantation may become a new method for treating ANFH. However, there are some limitations in the current study. Firstly, the BMSCs were not characterized by cell surface specific markers and their osteogenic/adipogenic differentiation potential was also not identified. However, in fact, the BMSCs used in the present study were just the same as those used in a prior study (16), which had demonstrated identification and characterization of BMSCs. Secondly, the femoral head necrosis negative control group and CD + BMSCs group were not included and analyzed in the current study. Thus, the experimental design appeared to be not rigorous enough. Therefore, further investigations should be undertaken to improve the above shortcomings.

In conclusion, good blood circulation and the osteogenic capability of the femoral head are key factors preventing the progression of ANFH. The results of the current study confirmed that the combination of decompression of the medullary center, removal of dead bone and implantation of BMSCs-nHAC/PLA scaffolds are conducive to regeneration and repair in cases of femoral head necrosis, and to the prevention of further necrosis. To some extent, BMSCs-nHAC/PLA enhances the therapeutic effect of CD on ANFH; combining the two may represent a novel treatment for femoral head necrosis.

\section{Acknowledgements}

Not applicable.

\section{Funding}

The current study was funded by the Science and Technology Key Projects Program of Shandong Province (grant no. 2009GG10002036).

\section{Availability of data and materials}

The datasets used and/or analyzed during the current study are available from the corresponding author on reasonable request.

\section{Authors' contributions}

DW and LX provided the experimental design and guidance. LW performed in vivo and in vitro experiments and drafted the manuscript. $\mathrm{CP}$ analyzed the data. $\mathrm{XX}$ identified and provided bone marrow mesenchymal stem cells from rabbits. YW conducted imaging examinations and analyses. GT carried out histological examinations and analyses.

\section{Ethics approval and consent to participate}

All operations involving laboratory animals were approved by the Animal Research Ethics Committee of the Second Hospital of Shandong University.

\section{Patient consent for publication}

Not applicable.

\section{Competing interests}

The authors declare that they have no competing interests.

\section{References}

1. Moya-Angeler J, Gianakos AL, Villa JC, Ni A and Lane JM: Current concepts on osteonecrosis of the femoral head. World J Orthop 6: 590-601, 2015.

2. van der Jagt D, Mokete L, Pietrzak J, Zalavras CG and Lieberman JR: Osteonecrosis of the femoral head: Evaluation and treatment. J Am Acad Orthop Surg 23: 69-70, 2015.

3. Mont MA, Jones LC and Hungerford DS: Nontraumatic osteonecrosis of the femoral head: Ten years later. J Bone Joint Surg Am 88: 1117-1132, 2006.

4. Ficat RP: Treatment of avascular necrosis of the femoral head. Hip 279-295, 1983.

5. Zhao DW, Yu M, Hu K, Wang W, Yang L, Wang BJ, Gao XH, Guo YM, Xu YQ, Wei YS, et al. Prevalence of nontraumatic osteonecrosis of the femoral head and its associated risk factors in the Chinese population: Results from a nationally representative survey. Chin Med J (Eng) 128: 2843-2850, 2015.

6. Larson E, Jones LC, Goodman SB, Koo KH and Cui Q: Early-stage osteonecrosis of the femoral head: Where are we and where are we going in year 2018? Int Orthop 42: 1723-1728, 2018.

7. Sultan AA, Khlopas A, Surace P, Samuel LT, Faour M, Sodhi N, Krebs VE, Stearns KL, Molloy RM and Mont MA: The use of non-vascularized bone grafts to treat osteonecrosis of the femoral head: Indications, techniques, and outcomes. Int Orthop 43: 1315-1320, 2019.

8. Wang Y, Kim UJ, Blasioli DJ, Kim HJ and Kaplan DL: In vitro cartilage tissue engineering with $3 \mathrm{D}$ porous aqueous-derived silk scaffolds and mesenchymal stem cells. Biomaterials 26: 7082-7094, 2005.

9. Fan L, Zhang C, Yu Z, Shi Z, Dang X and Wang K: Transplantation of hypoxia preconditioned bone marrow mesenchymal stem cells enhances angiogenesis and osteogenesis in rabbit femoral head osteonecrosis. Bone 81: 544-553, 2015.

10. Li D, Xie X, Yang Z, Wang C, Wei Z and Kang P: Enhanced bone defect repairing effects in glucocorticoid-induced osteonecrosis of the femoral head using a porous nano-lithium-hydroxyapatite/gelatin microsphere/erythropoietin composite scaffold. Biomater Sci 6: 519-537, 2018.

11. Lai Y, Cao H, Wang X, Chen S, Zhang M, Wang N, Yao Z, Dai Y, Xie X, Zhang P, et al: Porous composite scaffold incorporating osteogenic phytomolecule icariin for promoting skeletal regeneration in challenging osteonecrotic bone in rabbits. Biomaterials 153: 1-13, 2018.

12. Wu S, Xiao Z, Song J, Li M and Li W: Evaluation of BMP-2 enhances the osteoblast differentiation of human amnion mesenchymal stem cells seeded on nano-hydroxyapatite/Collagen/Poly(l-Lactide). Int J Mol Sci 19: pii E2171, 2018.

13. Wu DJ, Hao AH, Zhang C, Cui FZ, Wang XW, Gao CZ, Zhou CJ, Liu JL, Qiao Y and Ma SZ: Promoting of angiogenesis and osteogenesis in radial critical bone defect regions of rabbits with nano-hydroxyapatite/collagen/PLA scaffolds plus endothelial progenitor cells. Zhonghua Yi Xue Za Zhi 92: 1630-1634, 2012 (In Chinese). 
14. Rajagopal M, Balch Samora J and Ellis TJ: Efficacy of core decompression as treatment for osteonecrosis of the hip: A systematic review. Hip Int 22: 489-493, 2012.

15. He Z: The Guide for the Care and Use of Laboratory Animals. China Science Press, Washington, 2016.

16. Xie X, Du X, Li K, Chen Y, Guan Y, Zhao X, Niu G, Luan Y, Zhang D, Sun C, et al: Construction of engineered corpus cavernosum with primary mesenchymal stem cells in vitro. Sci Rep 7: 18053, 2017.

17. Wang X, Xing H, Zhang G, Wu X, Zou X, Feng L, Wang D, Li M, Zhao J, Du J, et al: Restoration of a critical mandibular bone defect using human alveolar bone-derived stem cells and porous nano-HA/Collagen/PLA scaffold. Stem Cells Int 2016: 8741641, 2016.

18. E LL, Xu WH, Feng L, Liu Y, Cai DQ, Wen N and Zheng WJ: Estrogen enhances the bone regeneration potential of periodontal ligament stem cells derived from osteoporotic rats and seeded on nano-hydroxyapatite/collagen/poly(L-lactide). Int J Mol Med 37: 1475-1486, 2016.

19. O'Brien FJ: Biomaterials \& scaffolds for tissue engineering. Materials Today 14: 88-95, 2011.

20. Kihara T, Hirose M, Oshima A and Ohgushi H: Exogenous type I collagen facilitates osteogenic differentiation and acts as a substrate for mineralization of rat marrow mesenchymal stem cells in vitro. Biochem Biophys Res Commun 341: 1029-1035, 2006.
21. Zhang C, Hu YY, Cui FZ, Zhang SM and Ruan DK: A study on a tissue-engineered bone using rhBMP-2 induced periosteal cells with a porous nano-hydroxyapatite/collagen/poly(L-lactic acid) scaffold. Biomed Mater 1: 56-62, 2006.

22. Griffith LG and Naughton G: Tissue engineering-current challenges and expanding opportunities. Science 295: 1009-1014, 2002.

23. Zhang HX, Zhang XP, Xiao GY, Hou Y, Cheng L, Si M, Wang SS, $\mathrm{Li} \mathrm{YH}$ and Nie L: In vitro and in vivo evaluation of calcium phosphate composite scaffolds containing BMP-VEGF loaded PLGA microspheres for the treatment of avascular necrosis of the femoral head. Mater Sci Eng C Mater Biol Appl 60: 298-307, 2016.

24. Fu Q, Tang NN, Zhang Q, Liu Y, Peng JC, Fang N, Yu LM, Liu JW and Zhang T: Preclinical study of cell therapy for osteonecrosis of the femoral head with allogenic peripheral blood-derived mesenchymal stem cells. Yonsei Med J 57: 1006-115, 2016.

25. Zhang YG, Wang X, Yang Z, Zhang H, Liu M, Qiu Y and Guo X: The therapeutic effect of negative pressure in treating femoral head necrosis in rabbits. PLoS One 8: e55745, 2013.

This work is licensed under a Creative Commons Attribution-NonCommercial-NoDerivatives 4.0 International (CC BY-NC-ND 4.0) License. 